\title{
Modeling of soft impingement effect during solid-state partitioning phase transformations in binary alloys
}

\author{
Hao Chen - Sybrand van der Zwaag
}

Received: 14 July 2010/Accepted: 13 September 2010/Published online: 23 September 2010

(C) The Author(s) 2010. This article is published with open access at Springerlink.com

\begin{abstract}
The soft impingement effect at the later stage of partitioning phase transformations has been modeled both for the diffusion-controlled growth model and for the mixed-mode model. Instead of the linear and exponential approximations for the concentration gradient in front of the interface used in the past, a general polynomial method of dealing with the diffusion field is proposed. The linear and exponential diffusion field approximations are two specific cases of the polynomial diffusion field approximation. The effect of soft impingement on the overall partitioning phase transformation is only related to the degree of the super-saturation in case of the diffusioncontrolled growth model, while it is determined by both the growth mode and the degree of super-saturation in case of the mixed-mode model.
\end{abstract}

\section{Introduction}

During the partitioning phase transformation, two simultaneous processes take place: diffusion of alloying elements ahead of the interface and migration of the interface. In the past, much research has been done to investigate the dominant process during the growth process of the partitioning phase transformation, and two classical models have been developed: the diffusion-controlled growth model [1] and the interface-controlled growth model [2]. Recently, a number of publications [3-5] have shown that the growth kinetics of the partitioning phase transformation cannot be described correctly by the classical models.

H. Chen $(\bowtie) \cdot$ S. van der Zwaag

Faculty of Aerospace Engineering, Delft University of

Technology, Kluyverweg 1, 2629 HS Delft, The Netherlands

e-mail: hao.chen@tudelft.nl
Consequently, a mixed-mode model [3-5] has been developed, which predicts that the growth mode of any partitioning phase transformation become more and more diffusion controlled as the phase transformation proceeds. The diffusion-controlled growth model and the interfacecontrolled growth model are the two extremes of the mixed-mode model.

Normally, the partitioning phase transformation can be divided into two stages [6-8]: (1) the first stage of the phase transformation in which the diffusion fields in front of opposing interfaces in the parent phase do not overlap; (2) the second stage in which the diffusion fields start to overlap, and the phase transformation slows down, the so-called soft impingement effect [6-8]. As diffusioncontrolled growth models have been proposed for a very long time, the analytical diffusion-controlled growth model for the first, non-overlapping diffusion fields stage have been well developed and are widely applied to describe the kinetics of phase transformation $[1,9,11]$. For the second stage, initially a so called mean field approximation [12] was used to take the soft impingement effect into account in the diffusion-controlled growth models. Later, to treat the overlap of diffusion filed in a more strict way, a number of diffusion-controlled growth models [7, 8, 13], assuming a linear diffusion field in front of the interface, have been developed to describe the soft impingement effect more accurately.

In [3], assuming a linear diffusion field in front of the interface, an analytical mixed-mode model has been developed to indicate the mixed-mode character of the partitioning phase transformation. However, a recent study by Bos and Sietsma [14] has shown that the original mixedmode model underestimates the partitioning phase transformation kinetics because of the linear diffusion field approximation. Also, the soft impingement effect at the 
later stage of partitioning phase transformations is not considered in the original mixed-mode model.

In this study, based on the polynomial method, a precise diffusion profile expression is introduced to reformulate the analytical diffusion-controlled growth model and the analytical mixed-mode model with considering soft impingement effect, and the newly reformulated analytical models are validated by a comparison with a fully numerical solution. Furthermore, the effect of soft impingement on the overall phase transformation kinetics is investigated for both the diffusion-controlled growth model and the mixedmode model, and results are compared.

\section{Analytical models}

Figure 1a and $\mathrm{b}$ are the schematics which illustrate the evolution of diffusion fields as the interface migrates from the $\alpha$-phase into the $\beta$-phase for the diffusion-controlled growth model and the mixed-mode model, respectively. The main difference between the diffusion-controlled growth model and the mixed-mode model is that the solute concentration at the interface in the diffusion-controlled growth model is assumed to be fixed during the entire phase transformation, while it should change physically in the mixed-mode model. Referring to Fig. 1, we define $t_{2}$ as the time after which the diffusion fields start to overlap, $x_{0}$ as the interface position, $L$ as the length of diffusion field, $C_{\mathrm{eq}}^{\beta \alpha}$ and $C_{\mathrm{eq}}^{\alpha \beta}$ as the equilibrium concentration in the $\beta$ - and $\alpha$-phase, $C_{0}$ as the bulk concentration, $C_{\mathrm{m}}$ as the carbon concentration at the center of the $\beta$-phase, and $2 X$ as the thickness of the parent phase.

\section{Diffusion-controlled growth model}

In the classical diffusion-controlled model for solid-solid partitioning phase transformations [1], local equilibrium is assumed to be maintained at the interface during the entire phase transformation, which means that chemical potential of all alloying elements is equal and there is no chemical Gibbs energy difference at the interface itself during the phase transformation. Local equilibrium can be maintained only when the interface mobility is infinitely fast.

\section{Non-overlapping diffusion stage}

When $x_{0} \leq x \leq x_{0}+L$, the diffusion profile in front of the interface as a function of position $x$ for the non-overlapping diffusion stage in the diffusion-controlled growth model is described in a quadratic form here:

$C(x)=A_{1}+A_{2}\left(x-x_{0}\right)+A_{3}\left(x-x_{0}\right)^{2}$
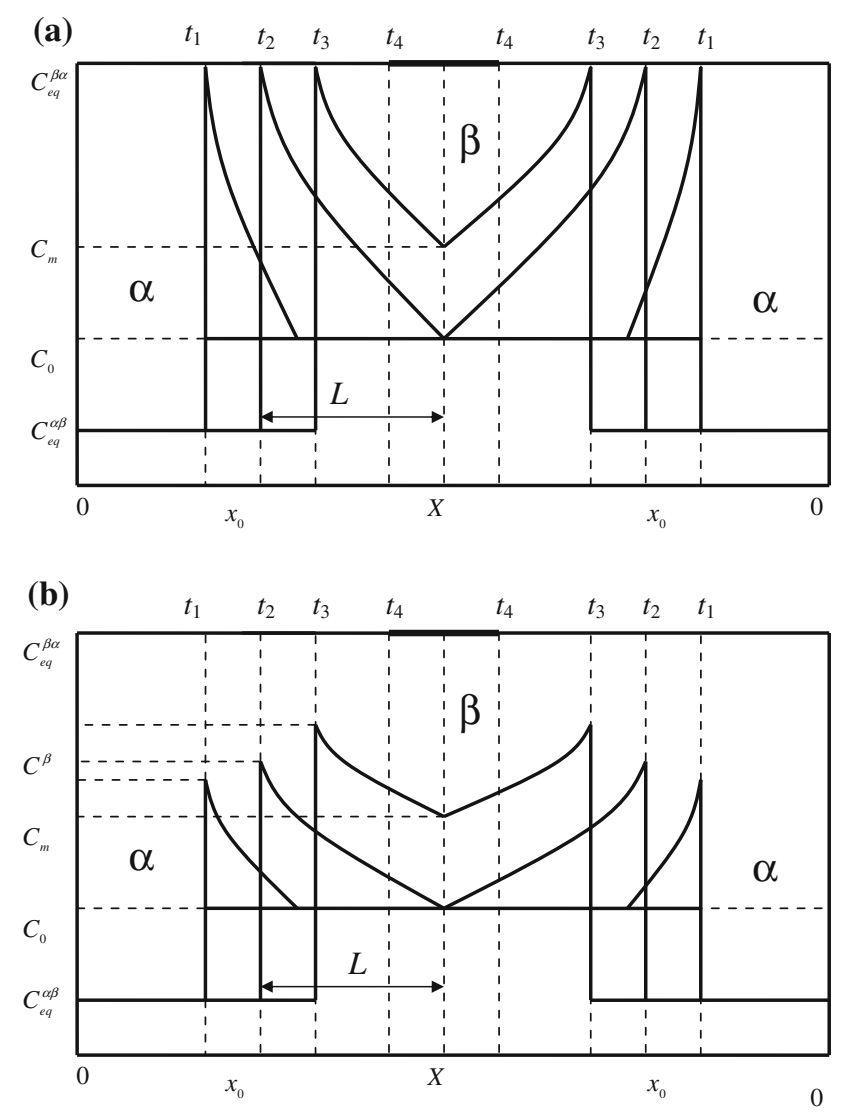

Fig. 1 The schematics of the diffusion fields evolution during the partitioning phase transformation for $\mathbf{a}$ the diffusion-controlled growth model and $\mathbf{b}$ the mixed-mode model

where $A_{1}, A_{2}$, and $A_{3}$ are the pre-factors, $C(x)$ is the solute concentration as a function of position.

The boundary conditions in the first stage can be described as

$C\left(x=x_{0}\right)=C_{\mathrm{eq}}^{\beta \alpha}$

$C\left(x=x_{0}+L\right)=C_{0}$

$\left.\frac{\partial C}{\partial x}\right|_{x=x_{0}+L}=0$

Based on the boundary conditions above, the pre-factors can be determined, and the quadratic diffusion profile for the non-overlapping diffusion stage can be written as

$C(x)=C_{0}+\left(C_{\text {eq }}^{\beta \alpha}-C_{0}\right)\left(1-\frac{x-x_{0}}{L}\right)^{2}, \quad x_{0} \leq x \leq x_{0}+L$

$C(x)=C_{0}, \quad x_{0}+L \leq x$

In order to obtain a general definition of the diffusion field ahead of the interface which encompasses both the linear and the quadratic type, the diffusion field is written here as 
$C(x)=C_{0}+\left(C_{\mathrm{eq}}^{\beta \alpha}-C_{0}\right)\left(1-\frac{x-x_{0}}{L}\right)^{n-1}, \quad n>1$

when $n=2$, it would become linear approximation as applied in [7, 13], when $n=3$, the diffusion field would be quadratic.

During the partitioning phase transformation, a mass balance law should always hold:

$$
\int_{0}^{x_{0}} C_{\mathrm{eq}}^{\alpha} \mathrm{d} x+\int_{x_{0}}^{x_{0}+L} C(x) \mathrm{d} x+\int_{x_{0}+L}^{X} C_{0} \mathrm{~d} x=\int_{0}^{X} C_{0} \mathrm{~d} x
$$

Combination of Eqs. 7 and 8 yields the expression for the diffusion length in front of the interface:

$L=\frac{n x_{0}\left(C_{0}-C_{\mathrm{eq}}^{\alpha \beta}\right)}{C_{\mathrm{eq}}^{\beta \alpha}-C_{0}}$

Since no accumulation of solutes will occur at the interface, the fluxes forwards and away from the interface should be equal, which can be expressed as

$v\left(C_{\mathrm{eq}}^{\beta \alpha}-C_{\mathrm{eq}}^{\alpha \beta}\right)=-D \frac{\partial C}{\partial x}$

Finally, the interface position as a function of time and the diffusion growth coefficient can be expressed as

$x_{0}=\lambda \sqrt{D t}$

$\lambda=\sqrt{\frac{2(n-1) \Omega^{2}}{n(1-\Omega)}}$

$\Omega=\frac{C_{\mathrm{eq}}^{\beta \alpha}-C_{0}}{C_{\mathrm{eq}}^{\beta \alpha}-C_{\mathrm{eq}}^{\alpha \beta}}$

where $\lambda$ is the diffusion growth coefficient, $\Omega$ is the degree of super-saturation.

\section{Overlapping diffusion stage}

In the overlapping diffusion stage, the diffusion profile in front of the interface and boundary conditions is written in the same form as Eqs. 1, 2, and 4, but the Eq. 3 is different and should be written as

$C\left(x=x_{0}+L\right)=C_{\mathrm{m}}$

The moment at which $C_{\mathrm{m}}$ starts to increase beyond $C_{0}$ is the start of soft impingement, and the $C_{\mathrm{m}}$ increases until the equilibrium concentration is approached at the final stage of partitioning phase transformation.

Similarly, the general polynomial diffusion profile for the overlapping diffusion stage can be written as
$C(x)=C_{\mathrm{m}}+\left(C_{\mathrm{eq}}^{\beta \alpha}-C_{\mathrm{m}}\right)\left(1-\frac{x-x_{0}}{L}\right)^{n-1}, \quad n>1$

The mass conservation law leads to

$\int_{0}^{x_{0}} C_{\mathrm{eq}}^{\alpha} \mathrm{d} x+\int_{x_{0}}^{X} C(x) \mathrm{d} x=\int_{0}^{X} C_{0} \mathrm{~d} x$

Combination of Eqs. 15 and 16 yields the expressions for the $C_{\mathrm{m}}$ and interface migration velocity:

$C_{\mathrm{m}}=\frac{1}{n-1}\left[\frac{n x_{0}}{L}\left(C_{0}-C_{\mathrm{eq}}^{\alpha \beta}\right)+n C_{0}-C_{\mathrm{eq}}^{\beta \alpha}\right]$

$v=D \frac{\left(C_{\mathrm{eq}}^{\beta \alpha}-C_{\mathrm{m}}\right)(n-1)}{\left(C_{\mathrm{eq}}^{\beta \alpha}-C_{\mathrm{eq}}^{\alpha \beta}\right) L}$

where the diffusion length $L\left(=X-x_{0}\right)$.

The mixed-mode model

In the mixed-mode model $[3,4]$, both the interface mobility and the finite diffusivity are considered to have effect on the kinetics of phase transformation, and the concentration of alloying elements at the interface does not evolve according to local equilibrium assumption but depends on the diffusion coefficient of alloying elements and interface mobility during the phase transformation. The mixed-mode model will also be reformulated in two stages here.

Non-overlapping diffusion stage

Generally, the interface velocity in the mixed-mode model can be written as [3]

$v=M \Delta G$

where $M$ is the effective interface mobility, $\Delta G$ is the driving force for interface migration and dependent on the solute concentration at the interface in the parent phase [3].

The interface mobility, $M$, which is temperaturedependent, can be expressed as

$M=M_{0} \exp \left(-Q_{G} / R T\right)$

where $M_{0}$ is a pre-exponential factor, $Q_{G}$ is the activation energy for the atomic motion.

The driving force, $\Delta G$, can be expressed as

$\Delta G=\sum_{i=1}^{p} C_{i}^{\alpha}\left(\mu_{i}^{\beta}-\mu_{i}^{\alpha}\right)$

where $p$ is the number of alloying elements in the system, $C_{i}^{\alpha}$ is the concentration of the alloying element $i$ in the 
$\alpha$-phase, $\mu_{i}^{\beta}$ and $\mu_{i}^{\alpha}$ are the chemical potential of the alloying element $i$ in the $\beta$ - and $\alpha$-phase, respectively.

In this study, only one alloying element will be considered, thus the driving force, $\Delta G$, can be approximated to be proportional to the deviation of the mobile alloying element concentration in the parent phase at the interface from the equilibrium concentration, and can be expressed as

$\Delta G=\chi\left(C_{\mathrm{eq}}^{\beta \alpha}-C^{\beta}\right)$

where $\chi$ is proportionality factors which can be calculated by Thermo-Calc and $C^{\beta}$ is the solute concentration at the interface in the $\beta$-phase

In the mixed-mode model, the diffusion profile in the first stage is still written in the polynomial way, the boundary conditions in the non-overlapping stage are expressed as

$C\left(x=x_{0}\right)=C^{\beta}$

$C\left(x=x_{0}+L\right)=C_{0}$

$\left.\frac{\partial C}{\partial x}\right|_{x=x_{0}}=0$

Based on the boundary conditions, the general polynomial diffusion profile for the first non-overlapping stage in the mixed-mode model can be derived as

$$
\begin{aligned}
& C(x)=C_{0}+\left(C^{\beta}-C_{0}\right)\left(1-\frac{x-x_{0}}{L}\right)^{n-1}, \\
& \quad x_{0} \leq x \leq x_{0}+L \\
& C(x)=C_{0}, \quad x_{0}+L \leq x
\end{aligned}
$$

Applying the mass balance law, the expression for the diffusion length can be obtained as

$L=\frac{n x_{0}\left(C_{0}-C_{\mathrm{eq}}^{\alpha \beta}\right)}{C^{\beta}-C_{0}}$

As there is no accumulation of solutes at the interface, the following equation can be derived for the mixed-mode model:

$v\left(C^{\beta}-C_{\mathrm{eq}}^{\alpha \beta}\right)=M \chi\left(C_{\mathrm{eq}}^{\beta \alpha}-C^{\beta}\right)\left(C^{\beta}-C_{\mathrm{eq}}^{\alpha \beta}\right)=-D \frac{\partial C}{\partial x}$

The solute concentration at the interface can be obtained by solving Eq. 29: where $\Delta C_{0}=C_{0}-C_{\mathrm{eq}}^{\alpha \beta}$ and $Z=\frac{D(n-1)}{M \chi n x_{0}}$

The equation for the interface concentration is in the same form as that in the original mixed-mode model in which a linear diffusion field is assumed, however, the parameter $Z$ in the original mixed-mode model is just one case of that present in this study. When $n=2$, the mixedmode model presented here is the same as the original mixed-mode model.

\section{Overlapping diffusion stage}

The original mixed-mode model does not take the effect of soft impingement into account, while this effect will be considered in this study. Appling the same method described in the diffusion-controlled growth model, the interface concentration can be derived as

$C^{\beta}$

$=\frac{\left(\left(C_{\mathrm{eq}}^{\alpha \beta}+C_{\mathrm{eq}}^{\beta \alpha}\right)-Z\right)+\sqrt{\left(\left(C_{\mathrm{eq}}^{\alpha \beta}+C_{\mathrm{eq}}^{\beta \alpha}\right)-Z\right)^{2}-4\left(C_{\mathrm{eq}}^{\alpha \beta} C_{\mathrm{eq}}^{\beta \alpha}-Z \frac{X C_{0}-x_{0} C_{\mathrm{eq}}^{\alpha \beta}}{X-x_{0}}\right)}}{2}$

with $Z=\frac{D n}{M \chi\left(X-x_{0}\right)}$

The solute concentration at the center of $\beta$-phase $C_{\mathrm{m}}$ can be written as

$C_{\mathrm{m}}=\frac{1}{n-1}\left[\frac{n x_{0}}{L}\left(C_{0}-C_{\mathrm{eq}}^{\alpha \beta}\right)+n C_{0}-C^{\beta}\right]$

\section{Numerical solutions}

In this study, the Murray-Landis method $[15,16]$ is chosen for the numerical solution, and the finite difference equation is written as

$$
\begin{aligned}
\frac{c_{i, j+1}-c_{i, j}}{\Delta t}= & D \frac{\left(c_{i-1, j}-2 c_{i, j}-c_{i+1, j}\right)}{\Delta x^{2}} \\
& +v \frac{n-i\left(c_{i+1, j}-c_{i-1, j}\right)}{n-1} 2 \Delta x
\end{aligned}
$$

where $c_{i}^{t}$ is the concentration at grid point $i$ at time $t, c_{i}^{t+\Delta t}$ is the concentration at grid point $i$ at time $t+\Delta t$. The second term on the right side of Eq. 33 accounts for the time dependence of the grid points. The diffusioncontrolled growth model and the mixed-mode model will

$$
C^{\beta}=\frac{\left(Z C_{0}+\Delta C_{0}\left(C_{\mathrm{eq}}^{\alpha \beta}+C_{\mathrm{eq}}^{\beta \alpha}\right)\right)+\sqrt{\left(Z C_{0}+\Delta C_{0}\left(C_{\mathrm{eq}}^{\alpha \beta}+C_{\mathrm{eq}}^{\beta \alpha}\right)\right)^{2}-\left(Z+2 \Delta C_{0}\right) \times\left(Z C_{0}^{2}+2 \Delta C_{0} C_{\mathrm{eq}}^{\alpha \beta} C_{\mathrm{eq}}^{\beta \alpha}\right)}}{\left(Z+2 \Delta C_{0}\right)}
$$


be combined with the Murray-Landis method to simulate the partitioning phase transformation here.

\section{Results and discussion}

To illustrate the effect of soft impingement here, the austenite to ferrite transformation in the binary $\mathrm{Fe}-1.0$ at. $\% \mathrm{C}$ alloys at $1050 \mathrm{~K}$ is investigated here. At the given temperature, Thermo-Calc gives $\chi=110 \mathrm{~J} /($ at. $\%)$, and the equilibrium carbon concentration in the austenite phase and ferrite phase is 2.05 and 0.09 at.\%, respectively. The diffusion coefficient of carbon in austenite is $1.14 \times$ $10^{-12} \mathrm{~m}^{2} / \mathrm{s}$, and the interface mobility $M$ is taken to be $5.4 \times 10^{-8} \mathrm{~mol} \mathrm{~m} / \mathrm{Js}$. In order to consider the phase transformation in a finite medium, the finite thickness of the austenite phase $2 X$ is assumed to be $20 \mu \mathrm{m}$, and the specific volumes of both the phases are taken equal.

In Fig. 2a, the thickness of ferrite phase is calculated as a function of time by the mixed-mode model with linear diffusion field and quadratic diffusion field. The solid line in the figure is the modeling results with soft impingement correction, and the dotted line is the results without soft
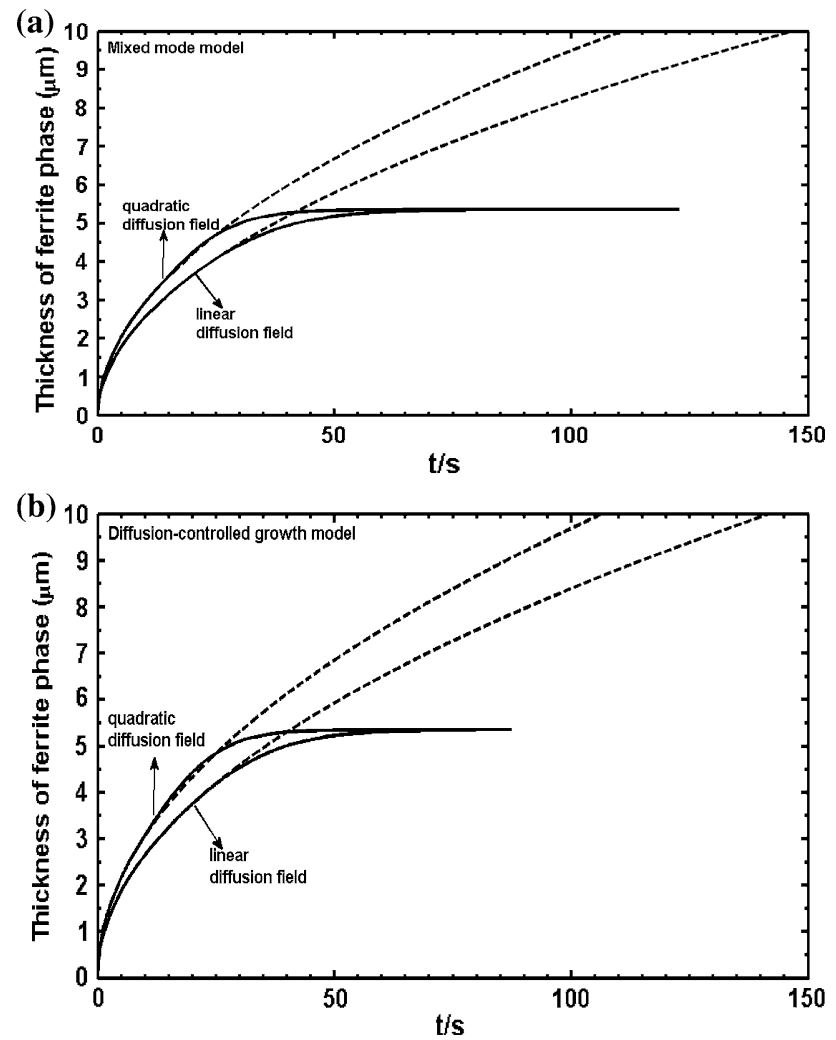

Fig. 2 The thickness of ferrite phase as a function of time during the austenite to ferrite transformation in $\mathrm{Fe}-1.0$ at. $\% \mathrm{C}$ at $T=1050 \mathrm{~K}$ predicted by $\mathbf{a}$ the mixed-mode model and $\mathbf{b}$ the diffusion-controlled growth model with linear and quadratic diffusion field approximations impingement correction. It is shown that the mixed-mode model with soft impingement correction predicts the interface migration stops when the fraction of ferrite reaches the equilibrium value, while the mixed-mode model without soft impingement correction shows that the interface migration will not stop in finite time. Furthermore, the figure indicates that the mixed-mode model with linear diffusion field predicts a slower kinetics than the model with quadratic diffusion field does. Figure $2 b$ shows the results predicted by the diffusion-controlled growth model, which indicates that the diffusion-controlled growth model with soft impingement correction also predicts the partitioning phase transformation to finish as the thermodynamic equilibrium is approached.

Figure 3 shows the fraction of ferrite as a function of time obtained by the analytical models and the fully numerical solutions. It shows that both the analytical mixed-mode model and the analytical diffusion-controlled model with a quadratic diffusion field predict the kinetics more precisely than those with linear diffusion field assumption. Comparing the present mixed-mode model assuming a quadratic diffusion profile with the mixedmode model assuming an exponential diffusion profile in [14], the derived equation for solute concentration at the interface during the non-overlapping diffusion field stage is the same in these two reformulated mixed-mode models. However, as mentioned above, the exponential diffusion profile is only valid in the infinite medium, while the quadratic diffusion profile can also be applied in the overlapping diffusion field stage, as shown in "Model". It has to be mentioned here that the quadratic diffusion profile is just one case of polynomial diffusion profiles; in general, the exact diffusion profile can be approximated as

$$
\begin{gathered}
C(x)=A_{1}+A_{2}\left(x-x_{0}\right)+A_{3}\left(x-x_{0}\right)^{2}+\cdots+A_{n}\left(x-x_{0}\right)^{n-1}, \\
n=2,3,4 \ldots
\end{gathered}
$$

Equation 34 is a $(n-1)$ order polynomial diffusion profile, in which $n$ parameters have to be determined, which means $n$ boundary conditions are needed to solve the problem. Except Eqs. 2 and 3, $(n-2)$ extra boundary conditions can be written as

$\left.\frac{\partial^{m} C}{\partial x^{m}}\right|_{x=x_{0}+L}=0, \quad m=1,2, \ldots, n-2$

Based on the above boundary conditions, the parameters in Eq. 34 can be obtained. Although the accuracy of the model could possibly be increased increasing the order of the diffusion profile, it also make the case more complicated, that is why the linear diffusion profile [3, 7, 13] has been widely applied in the past. As shown in Fig. 3, the accuracy of the quadratic diffusion profile is quite close to that of the numerical solution, which means the accuracy will not be further improved significantly by increasing the 



Fig. 3 The fraction of ferrite as a function of time during the austenite to ferrite transformation in $\mathrm{Fe}-1.0$ at. $\% \mathrm{C}$ at $T=1050 \mathrm{~K}$ predicted by $\mathbf{a}$ the mixed-mode model and $\mathbf{b}$ the analytical model

order of the diffusion profile. Therefore, the quadratic diffusion field approximation would be applied to the analytical models in all the following calculations.

Considering the soft impingement effect at the later stage, the solute concentration at the center of the austenite phase and diffusion length, which are the two key parameters in the overlapping diffusion stage, is calculated as a function of time by the mixed-mode model and diffusioncontrolled growth model in Fig. 4a and b, respectively. The vertical dotted line in the figure indicates the critical point at which the diffusion fields in the neighboring grains start to overlap. Before the critical point is reached, the diffusion length extends as the interface migrates into the austenite phase, and the solute concentration at the center of the austenite phase is not affected by diffusion and is fixed at the bulk concentration of the $\mathrm{Fe}-\mathrm{C}$ alloys. After the critical point, the diffusion length would starts to shrink, and the solute concentration at the center of the austenite phase would begin to increase toward the equilibrium concentration. In the model considering the soft impingement, both the solute concentration at the center of the austenite phase and the diffusion length can be used to
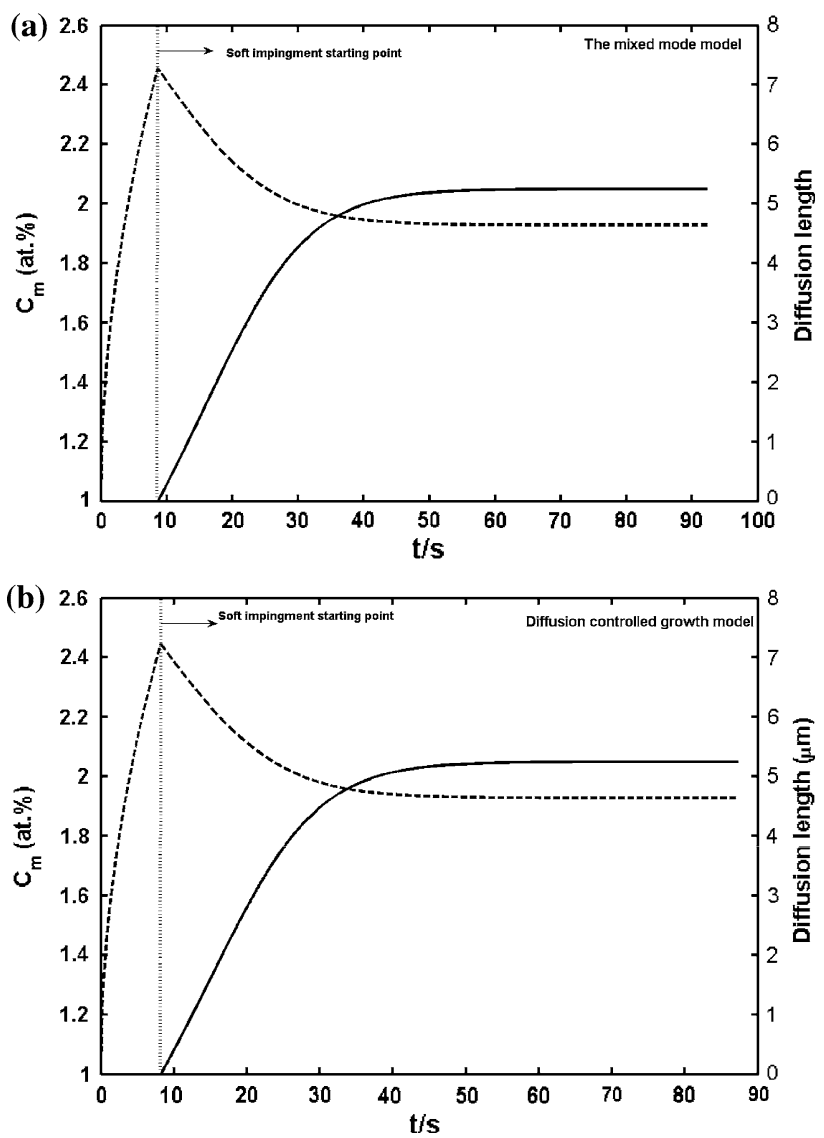

Fig. 4 The solute concentration at the center of the austenite phase and the diffusion length as a function of time predicted by a the mixed-mode model and $\mathbf{b}$ the diffusion-controlled growth model

estimate the soft impingement starting point, while only the diffusion length can be the effective factor for detecting soft impingement in the model without soft impingement correction.

As discussed in the original mixed-mode model [3], the bulk concentration $C_{0}$ affects the evolution of diffusion length during the austenite to ferrite phase transformation at a certain temperature, and it is concluded that the smaller the bulk concentration $C_{0}$, the shorter the diffusion length. However, at different temperatures, the equilibrium concentrations in the austenite and ferrite phase are different, which also affects the diffusion length evolution. Therefore, it is necessary and meaningful to summarize all the effect factors into one factor to obtain a general law. In Fig. 5, the diffusion lengths as a function of the thickness of ferrite phase for different degrees of super-saturation are calculated by the diffusion-controlled growth model. The value of diffusion length is only affected by the degree of super-saturation, in which the bulk concentration and the equilibrium concentration in both the austenite and the ferrite phase are included. It shows that the magnitude of the diffusion length decreases with increasing the degree 


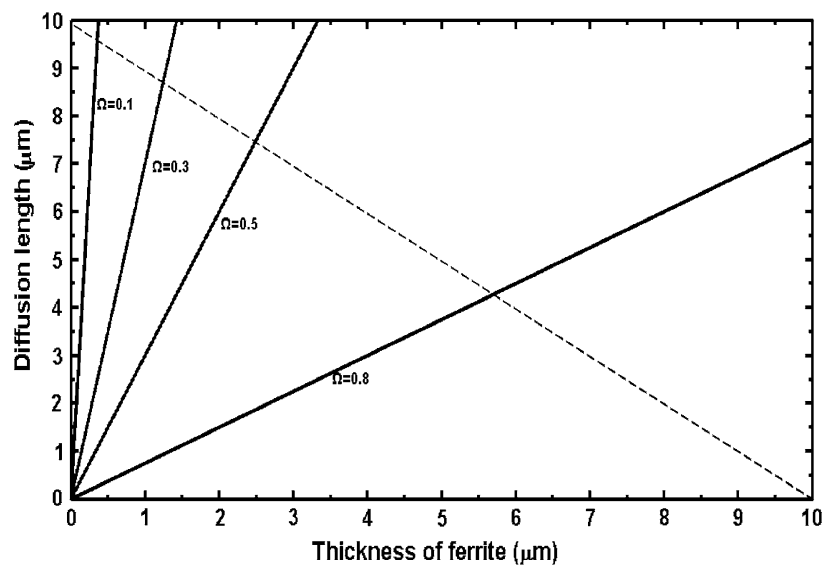

Fig. 5 The diffusion length as a function of the thickness of ferrite phase for different degrees of super-saturation predicted by the diffusion-controlled growth model, the dotted line indicates the start of soft impingement

of super-saturation. Actually, decreasing the bulk concentration at a certain temperature discussed in the original mixed-mode model [3] is just one specific case of increasing the degree of super-saturation according to Eq. 13, and Fig. 5 can be considered as a master curve for estimating the diffusion length.

In order to investigate whether the overlapping diffusion stage or the non-overlapping diffusion stage dominates the transformation kinetics, the ratio of ferrite transformed during the overlapping diffusion stage and the total equilibrium fraction of ferrite as a function of the degree of super-saturation is calculated by the diffusion-controlled growth model in Fig. 6. It is indicated that the ferrite transformed during the overlapping diffusion stage decreases with increasing the degree of super-saturation. This can be easily understood in this way: as the super-saturation

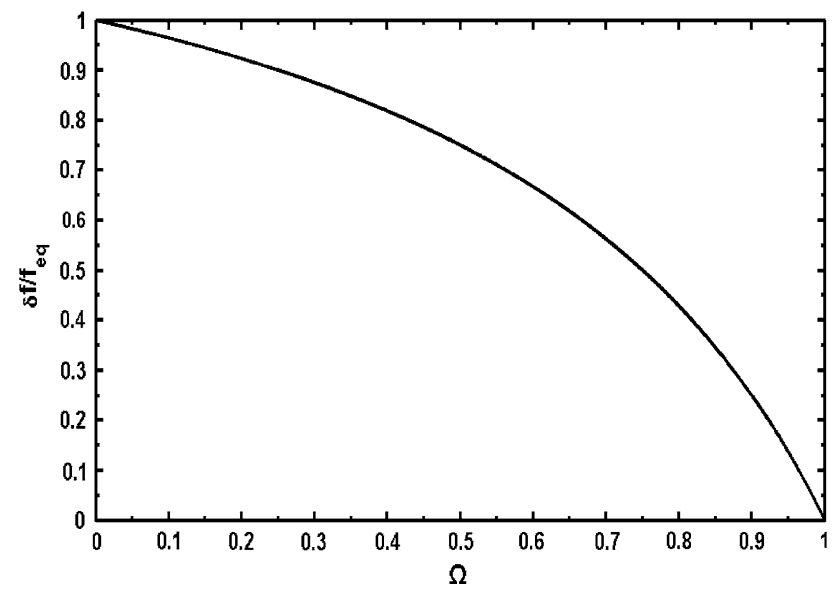

Fig. 6 The ratio of ferrite transformed during the overlapping diffusion stage and the equilibrium fraction of ferrite as a function of the degree of super-saturation predicted by the diffusion-controlled growth model increases, the solute concentration difference between the growing ferrite phase and the bulk concentration would be smaller, which means less carbon has to be rejected from the ferrite phase into the austenite phase as the interface migrates into the austenite phase and thus less carbon would pile up in front of the interface, so the overlapping diffusion stage would be shorter.

As shown in Figs. 5 and 6, the diffusion length in the diffusion-controlled growth model is a simple function of the degree of super-saturation. The magnitude of diffusion length in the mixed-mode model is not just determined by the degree of super-saturation but also by the diffusion coefficient and the interface mobility. As discussed in recent study [17], the ratio of the interface mobility and the diffusion coefficient has a strong effect on the kinetics of partitioning phase transformation, thus there is no doubt that the soft impingement effect during the partitioning phase transformation is also determined by this ratio. Compared with the diffusion coefficient, the value of interface mobility during partitioning phase transformation is still not known accurately [18-20]. Therefore, fixing the value of diffusion coefficient and varying the value of interface mobility, in Fig. 7, the diffusion length and size of ferrite phase during the austenite to ferrite phase transformation at $1050 \mathrm{~K}$ in $\mathrm{Fe}-1.0$ at.\% $\mathrm{C}$ alloys as a function of time are predicted by the mixed-mode model and the diffusion-controlled growth model. As the temperature and bulk concentration of the $\mathrm{Fe}-\mathrm{C}$ alloys are fixed, the degree of super-saturation is a constant, thus only the effect of interface mobility and diffusion coefficient on the soft impingement is investigated in Fig. 7. It is indicated that the increase in the interface mobility has a small effect on the diffusion length as a function of time but this leads to a significant increase in the interface migration rate, which would make the soft impingement start earlier. Also,

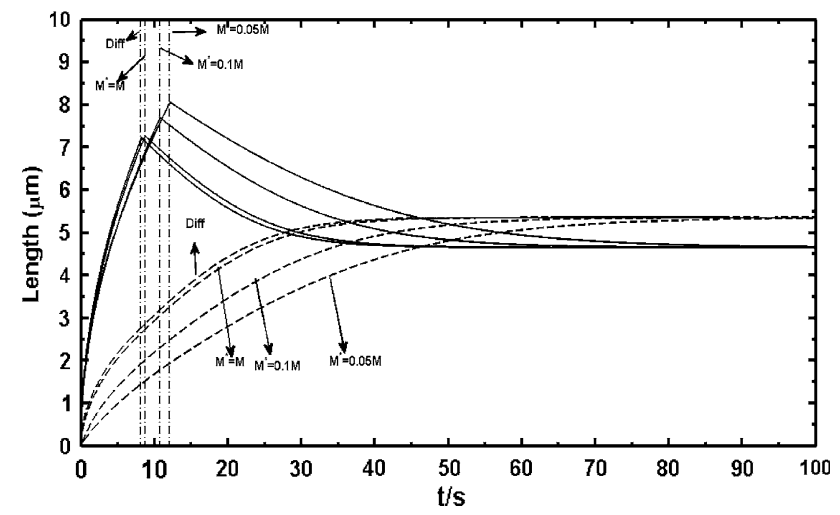

Fig. 7 The diffusion length (solid line) and size of ferrite (dotted line) as a function of time during the austenite to ferrite phase transformation at $1050 \mathrm{~K}$ in $\mathrm{Fe}-1.0$ at.\% $\mathrm{C}$ alloys predicted by the mixed-mode model with different interface mobilities and diffusioncontrolled growth model. Diff diffusion-controlled growth model 


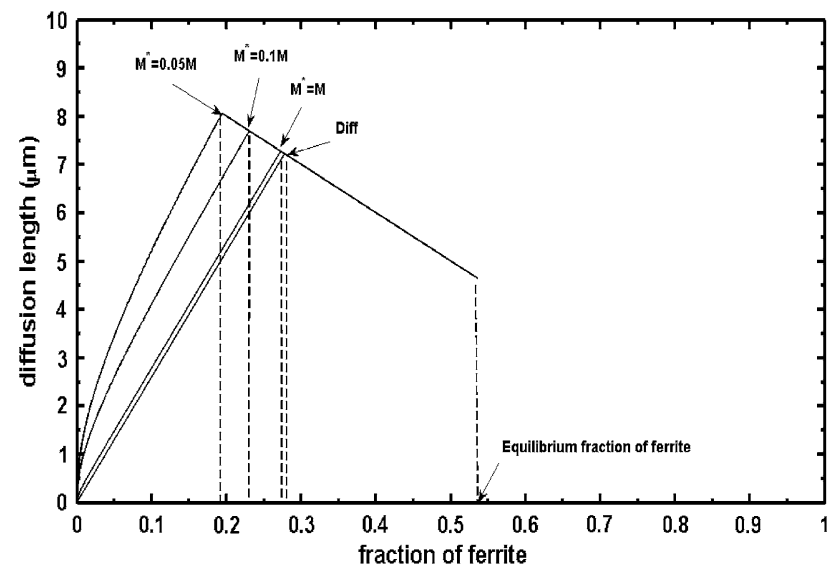

Fig. 8 The diffusion length as a function of the fraction of ferrite phase during the austenite to ferrite phase transformation at $1050 \mathrm{~K}$ in $\mathrm{Fe}-1.0$ at. $\% \mathrm{C}$ alloys predicted by the mixed-mode model with different interface mobilities and diffusion-controlled growth model. Diff diffusion-controlled growth model

increasing the interface mobility, the kinetics predicted by the mixed-mode model would become closer to that by the diffusion-controlled growth model, since the transformation kinetics is more and more controlled by the solutes diffusion, more discussion about this point can be found in [17]. In Fig. 8, the diffusion length as a function of the

(a)

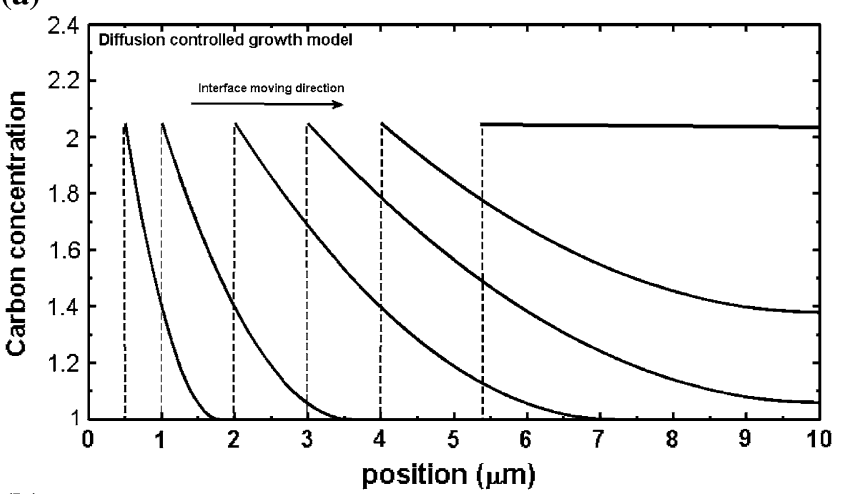

(b)

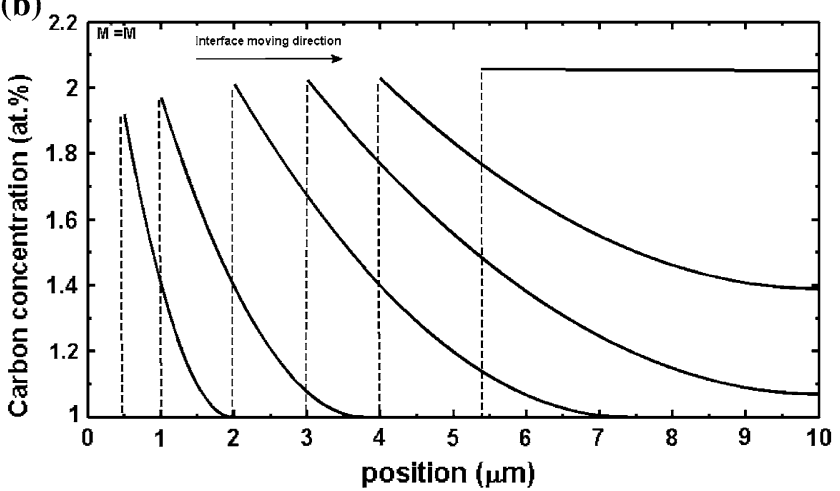

fraction of ferrite phase is calculated by the mixed-mode model with different interface mobilities and diffusioncontrolled growth model. Although the soft impingement starts earlier with increasing interface mobility as shown in Fig. 7, the ferrite transformed during the soft impingement stage decreases as shown in Fig. 8, which means the dominance of the soft impingement effect on the overall transformation kinetics reduces. In order to indicate the effect of interface mobility on the carbon profile evolution, in Fig. 9, the carbon profile in the austenite phase is calculated by the diffusion-controlled growth model and the mixed-mode model with different interface mobilities.

The carbon concentration at the interface in the austenite phase, which determines the magnitude of driving force for interface migration, is a key physical parameter in the mixed mode, thus it is calculated by the mixed-mode model in Fig. 10a. It shows that the interface concentration increases as the interface migrates, and the driving force would decrease to 0 and interface stops migrating when the equilibrium concentration is approached. The interface concentration predicted by the mixed-mode model without soft impingement correction [14] would not be equal to equilibrium concentration even when the equilibrium fraction is approached. It should be pointed out the interface concentration equals $C_{0}$ for all values of $M_{0}$ when the (c)
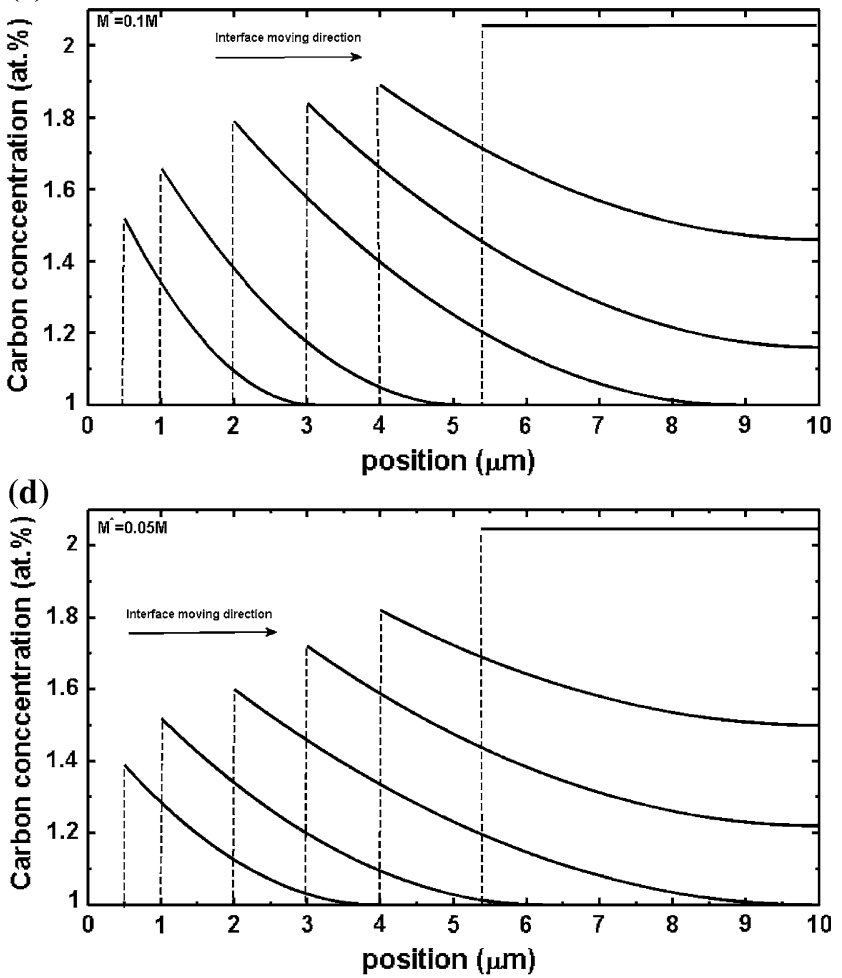

Fig. 9 The carbon profile evolution in the austenite phase predicted by the diffusion-controlled growth model and the mixed-mode model with different interface mobilities 


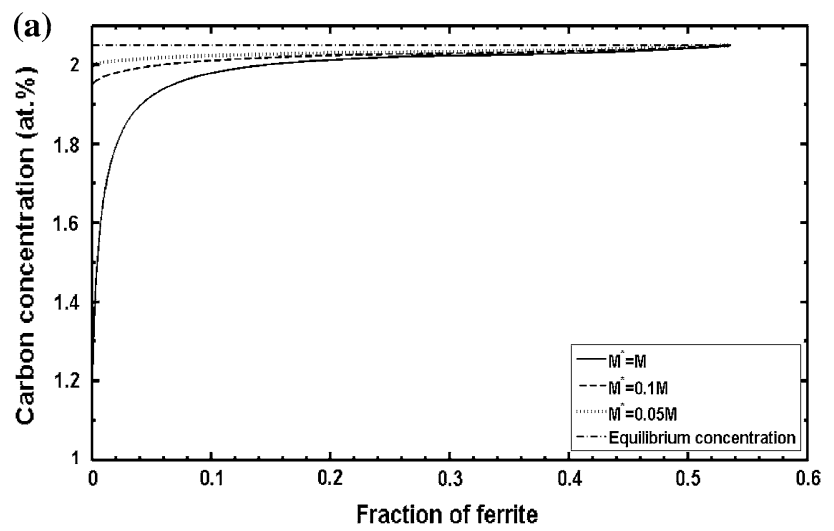

(b)

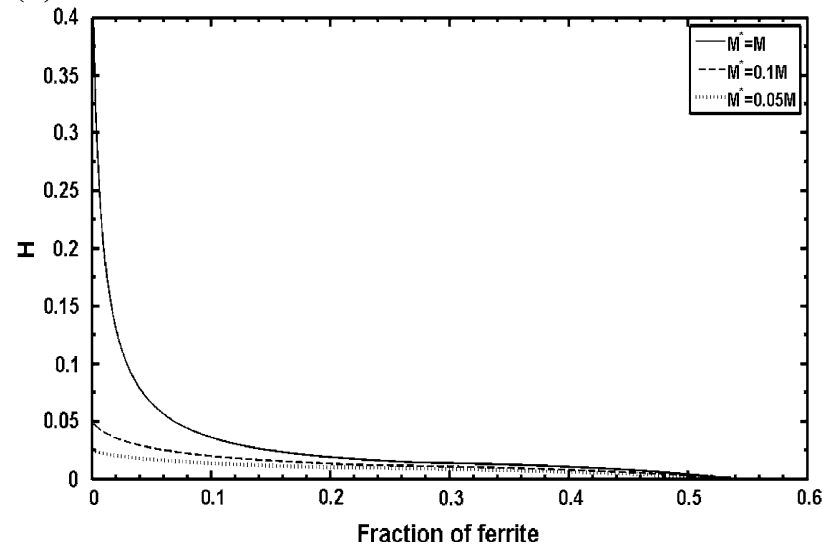

Fig. 10 a The carbon concentration at the interface and $\mathbf{b}$ the growth mode $H$ as a function of the fraction of ferrite predicted by the mixedmode model

ferrite fraction is zero. However, in the initial stage, the interfacial carbon concentration increase extremely fast, this steep increase is not properly reflected in Fig. 10a. In Fig. 10b, the newly defined growth mode parameter $H\left(=\frac{C_{\mathrm{eq}}^{\beta \alpha}-C^{\beta}}{C_{\mathrm{eq}}^{\beta \alpha}-C_{\mathrm{eq}}^{\alpha \beta}}\right)[17]$ as a function of fraction of ferrite phase for different interface mobilities is presented. For a certain interface mobility, the $H$ decreases as the phase transformation proceeds, which indicates that the growth mode become more diffusion controlled. As the soft impingement effect is corrected in the present mixed-mode model, the growth mode parameter would decrease to 0 when the thermodynamic equilibrium is established. Also, the growth mode is changed correspondingly with the variation of interface mobility, and the growth mode is approaching to the pure diffusion-controlled growth with increasing the ratio of interface mobility and the diffusion coefficient. In other words, it can also be stated that the dominance of soft impingement on the transformation kinetics is determined by the growth mode if the degree of super-saturation is fixed.

\section{Conclusions}

Applying the polynomial diffusion field and considering the diffusion field overlap quantitatively at the later stage of phase transformation, the mixed-mode model and diffusion-controlled growth model are reformulated in an analytical form to be more accurate and physically reasonable in this study. The effect of soft impingement on the overall partitioning phase transformation kinetics is solely determined by the super-saturation according to diffusioncontrolled growth, and it decreases with increasing the super-saturation. However, in the mixed-mode model, the effect of soft impingement on the overall phase transformation kinetics is determined by both the degree of the super-saturation and the growth mode.

Open Access This article is distributed under the terms of the Creative Commons Attribution Noncommercial License which permits any noncommercial use, distribution, and reproduction in any medium, provided the original author(s) and source are credited.

\section{References}

1. Zener C (1949) J Appl Phys 20:950

2. Christian JW (2003) Theory of transformations in metal and alloys, part I. Pergamon Press, Oxford

3. Sietsma J, van der Zwaag S (2004) Acta Mater 52:4143

4. Krielaart GP, Sietsma J, van der Zwaag S (1997) Mater Sci Eng A $237: 216$

5. Huizenga RM, Bos C, Sietsma J (2008) J Mater Sci 43:3744. doi:10.1007/s10853-007-2219-0

6. Wert C, Zener C (1950) J Appl Phys 21:5

7. Fan K, Liu F, Liu XN, Zhang YX, Yang GC, Zhou YH (2008) Acta Mater 56:4309

8. Offerman SE, van Dijk NH, Sietsma J, Lauridsen EM, Margulies L, Grigull S, Poulsen HF, van der Zwaag S (2004) Acta Mater $52: 4757$

9. Offerman SE, van Dijk NH, Sietsma J, Grigull S, Lauridsen EM, Margulies L, Poulsen HF, Rekveldt MT, van der Zwaag S (2002) Science 298:1003

10. Schmidt ED, Damm EB, Sridhar S (2007) Metall Mater Trans A 38A:698

11. Chen H, Liu YC, Yan ZS, Li YL, Zhang LF (2010) Appl Phys A 98:211

12. Crespo D, Pradell T, ClavagueraMora MT, Clavaguera N (1997) Phys Rev B 55:3435

13. Wang HF, Liu F, Zhang T, Yang GC, Zhou YH (2009) Acta Mater 57:3072

14. Bos C, Sietsma J (2007) Scripta Mater 57:1085

15. Vander Ven A, Delaey L (1996) Prog Mater Sci 40:181

16. Pernach M, Pietrzyk M (2008) Comput Mater Sci 44:783

17. Chen H, van der Zwaag S (2010) Comput Mater Sci 49:801

18. Strandlund H, Odqvist J, Agren J (2008) Comput Mater Sci 44:265

19. Hillert M, Hoglund L (2006) Scripta Mater 54:1259

20. Gamsjager E, Militzer M, Fazeli F, Svoboda J, Fisher FD (2006) Comput Mater Sci 37:94 